
\title{
$\begin{array}{ll}\text { Research Square } & \begin{array}{l}\text { Preprints are preliminary reports that have not undergone peer review. } \\ \text { They should not be considered conclusive, used to inform clinical practice, } \\ \text { or referenced by the media as validated information. }\end{array}\end{array}$
}

\section{The unexplained increasing of Erythrocyte Sedimentation Rate in a case recovered from COVID-19}

\section{Shenglan Pu}

1. Department of General Medicine, First People's Hospital of Zunyi; 2. Third Affiliated Hospital of Zunyi Medical University

\section{Xiangyan Zhang}

1.Respiratory Institute, Guizhou Provincial People's Hospital; 2. NHC Key Laboratory of Pulmonary Immune related Disease ( Guizhou Provincial People's Hospital,GZU)

\section{Daishun Liu}

1.Respiratory Departments, First People's Hospital of Zunyi; 2.Third Affiliated Hospital of Zunyi Medical University

\section{Baning Ye}

Intensive Care Unit, Guizhou provincial people's Hospital.

Jianquan Li ( $2401131098 @ q q . c o m)$

1. Intensive Care Unit, Guizhou provincial people's Hospital; 2. NHC Key Laboratory of Pulmonary Immune related Disease ( Guizhou Provincial People's Hospital,GZU)

\section{Case Report}

Keywords: COVID-19, Erythrocyte Sedimentation Rate, Prognosis, Case report

Posted Date: April 7th, 2020

DOI: https://doi.org/10.21203/rs.3.rs-21001/v1

License: (c) (i) This work is licensed under a Creative Commons Attribution 4.0 International License. Read Full License 


\section{Abstract}

Background A novel coronavirus virus pneumonia (named COVID-19) broke out in Wuhan, China on December 2019, and spread around of the word [1, 2]. As of March $4^{\text {th }}, 2020,93090$ confirmed cases and 2984 deaths have been reported in more than 80 countries and territories. It has triggered global public health security $[2,3]$. However, the features and prognosis of COVID-19 are incompletely understood [2].

Case presentation We here report that the erythrocyte sedimentation rate (ESR) increased in a confirmed COVID patient, the high level of ESR sustained for long time even patient recovered from COVID-19, while all results related to tumor, tuberculosis, rheumatic diseases, anemia, et al. cannot explain the abnormal ESR presented in this case.

Conclusion Although the increased ESR cannot be explained by all existing evidences, which possibly links the abnormal pathologic change in some COVID-19 patients and negative prognosis, and provides the clue to dissect the mechanism of illness progressing in COVID-19 and its prognosis.

\section{Background}

In December, 2019, a cluster of patients with pneumonia of unknown cause emerged in Wuhan, China, and later were confirmed with infection of a novel coronavirus virus (named SARS-CoV-2). Although the initial signs and symptoms for most COVID-19 patients presented as fever or cough [4], the symptoms of extra-pulmonary also had been reported [5]. Recent research has shown that patient with no any clinical signs could be infected by the SARS-CoV-2 [6], while other studies have shown the COVID-19 can rapidly evolved into the acute respiratory distress syndrome (ARDS) even multiple organ dysfunction (MODS) [4] [7]. Due to most researches focused the changes of respiratory system, thus so far our understanding to COVID-19 is still superficial, the features of other organ involvement and prognosis in COVID-19 patients are unclear.

We describe an unexplained phenomenon that the high level of ESR presented in a case of COVID-19, and aim to dissect the cause of abnormal ESR in this case, by which provide the insight to understand the illness progression, and long-term impact of COVID-19 on patients.

\section{Case Presentation}

A 51-year-old male patient residing in Guizhou Province, China returned from Wuhan, China on January 22,2020 where there had been an outbreak of COVID-19 pneumonia. He presented the 5 years of history of hypertension but denied the history of arthritis, hyperthyroidism, tuberculosis, autoimmune disease and other chronic diseases. He was admitted to Guizhou Provincial People's Hospital on January 29, 2020 with high fever for 8 days, additional clinical symptoms included dry cough, malaise and pronounced tiredness. On admission, physical examinations revealed a temperature of $37.8^{\circ} \mathrm{C}$, no evidence of lymphadenopathy, or physical signs suggestive of leukemia and hyperthyroidism. Detailed systemic examinations revealed no abnormalities in cardiovascular, or abdominal systems. Laboratory 
data included the mildly abnormal of blood routine examination and other inflammation parameters such as C-reactive protein (CRP), procalcitonin (PCT), interleukin-6 (IL-6) and ESR, normal live and renal function parameter (Table 1); Arterial blood gas analysis was as follows: $\mathrm{pH}, 7.418 ; \mathrm{PO} 2,71$ (oxygen Index 173); $\mathrm{PCO} 2,42 \mathrm{mmHg}$ on room air. Chest computed tomography $\mathbb{C} \mathrm{CT} \otimes$ showed the feature of multiple ground glass opacification in both lower lobes (Figure 1). Considering his epidemiological history traveled to Wuhan and sign of fever and dry cough, the nucleic acid assay of COVID-19 of throat swab was tested on January 29 and 30,2020. Combined the twice positive results of nucleic acid assay of COVID19 , his symptoms and laboratory data, the COVID-19 pneumonia was confirmed on January 31,2020 according to the diagnostic criteria for COVID-19 pneumonia.

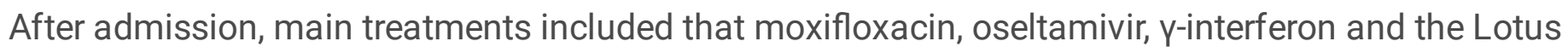
antipyretic granules. As to February 02, 2020, he still had the signs of intermittent fever and dry cough, therefore, the anti-virus drug, Lopinavir and Ritonavir Tablets, the methylprednisolone were given. Up to February 14,2020 , although patient's symptoms of malaise and pronounced tiredness were significantly improved, he still had the dry cough and intermittent fever. For centralized management to COVID-19 patients, he was transferred to Jiangjunshan Hospital (The Fixed Hospital for COVID-19 patients in Guizhou Province, China). Laboratory data examined after transferring indicated the count of leukocyte and lymphopenia decreased, while IL -6 and CRP and ESR increased $\nabla$ and the mildly abnormal liver function (Table 2), Arterial blood gas analysis was as follows: pH, 7.41 PO2, 78(oxygen Index 270); PCO2, $42 \mathrm{mmHg}$ on room air (Table 4). The chest CT showed the streaky or coarse reticular pattern opacities, and lesions in both lower lobes were improved comparing to that scanned before (Figure 2). Treatments with moxifloxacin and oseltamivir were replaced with the anti-virus drug, Arbido.

Up to February 21,2020 , the sign of dry cough and fever significantly removed. The examination of chest CT showed the lesions in both lower lobes significantly improved (Figure 3), and the assay of blood sample indicated all parameters of inflammation were normal except ESR (Table

3 ) tested on February 25, 2020. Also both the nucleic acid assay of throat swab of virus of COVID-19 tested on February 22 and 24, 2020 were negative, all which indicated this patient recovered from COVID-19.

However, the ESR gradually increased after admission, and reached at $120 \mathrm{~mm} / \mathrm{h}$ on February 22, 2020, to figure out the possible reasons increasing the ESR in this case, we tested the related parameters of tumor, inflammation, tuberculosis, rheumatic diseases, autoimmune diseases, hyperthyroidism and anemia, results showed there were no evidences indicated the increasing of ESR resulted from these possible factors (Table 4). With the improvement of patient's condition, we stopped the use of all antivirus on February 24,2020, patients situation was stable from February 24, 2020 to March 01, 2020, and the patient was discharged and entered the period of medical observation on March 1, 2020, the examination of ESR on February 28 and 29, 2020 still reached to $120 \mathrm{~mm} / \mathrm{h}$ and $118 \mathrm{~mm} / \mathrm{h}$ respectively (Figure 4), therefore we excluded the increasing of ESR resulted from the negative effect of drugs, but the exact cause of ESR increased in this case is unclear. 


\section{Discussion And Conclusion}

As its high susceptibility, COVID-19 caused the serious public heath event around of the world $[3,8]$. Although the treatments and prevention to COVID-19 pneumonia progressed, little is known about the prognosis and clinical impact of COVID-19 pneumonia $[2,9]$.

In this virus-imported case, the fever is the typical clinical feature of COVID-19 infection even though several inflammation parameters such as the count of leukocyte and neutrophils were normal (As shown in table

1). Consistent with common characteristics of viral infection, the abnormal of lymphopenia count presented after COVID-19 and returned to normal level after recovery from virus infection (As shown in table 1 and 3). With the illness progression, COVID-19 pneumonia could be associated with bacterial infection, as the results showed the parameters of inflammation such as the count of leukocyte and neutrophils, IL-6 and CRP increased. But the PCT results indicated the viral infection rather than bacterial infection mainly involved in this case (As shown in table 1-3). As the nucleic acid assay of virus turned negative, the patient's physical sign of fever and dry cough significantly removed, therefore, the signs and symptoms of the patient are correlated with the activity of the SARS-CoV- 2 .

Strangely, we found one unexplained laboratory data in this case that ESR began to significantly increase at about two weeks after COVID-19 infection, the high level of ESR sustained for long time even though the sign of fever and dry cough removed, the change of chest CT scanning improved (Shown in figure 3), and the test of nucleic acid assay of throat swab of virus of COVID-19 turned negative. While current results indicated the abnormal of ESR in this case was not caused by the tumor, inflammation, tuberculosis, rheumatic diseases hyperthyroidism, anemia, autoimmune diseases and the side-effect of drugs ( As shown in table 4).

The ESR is affected by the size, shape and concentration of red blood cells and plasma characteristics [10]. To date, the exact causes of the increased ESR in this case is not yet clear, the COVID-19 could trigger the change of the form of erythrocyte or plasma characteristics including the immune system by an unknown mechanism to increase the ESR. The sustained high level of ESR possibly brings the negative effect on COVID-19 patient's prognosis, for example, the high ESR could damage the joint, which leads to the joint disease such as osteoarthritis [11, 12]. Furthermore, it may be a precursor of hepatic and renal dysfunction [12-15]. Thus COVID-19 could influence the long-term prognosis of patient, although more cases and evidences need to be provided.

\section{Abbreviations}

Coronavirus Disease 2019

2019-novel coronavirus
COVID-19

2019-nCoV 
Erythrocyte sedimentation rate

Acute respiratory distress syndrome

Multiple organ dysfunction

C-reactive Protein

Procalcitonin

Interleukin-6

Chest computed tomography
ESR

ARDS

MODS

CRP

PCT

IL-6

CT

\section{Declarations}

Ethics approval and consent to participate

This retrospective study was conducted according to the ethical guidelines of the Helsinki Declaration and was approved by the Human Ethics Committee of Guizhou Provincial People's Hospital. Written informed consent was obtained from individual participants.

Consent for publication

Written informed consent was obtained from the patient for publication of this case report and any accompanying images. A copy of the written consent is available for review by the Editor of this journal.

Availability of data and material

We confirm that all data generated or analyzed during this study are included in this published article.

Competing interests

We confirm that the manuscript has been read and approved by all named authors, and the order of authors listed in the manuscript has been approved by all of us. All authors declare that they have no competing interests. 
Funding

This study was supported by the Central Public-interest Scientific Institution Basal Research Fund, Chinese Academy of Medical Sciences (CN) (2019PT320003). This Fund supported the protective clothing, gloves, goggles and N95 masks for Dr. Pu to enter the ward to collect the clinical data.

Authors' contributions

SP collected and interpreted the patient's clinical data regarding COVID-19. XZ and DL collected and analyzed all laboratory data in this case. BY contributed to the making of tables and figures. JL analyzed all data and contributed to the writing of manuscript. All authors read and approved the final manuscript. $\mathrm{JL}$ is responsible for communicating with the other authors about progress, submissions of revisions and final approval of proofs.

Acknowledgements

We thank Dr. Tingting Lou and Dr. Wenqi Zheng at Jianjunshan Hospital for their assistance in this preparation and collection of clinical data.

\section{References}

1. Zhu, N., et al., A Novel Coronavirus from Patients with Pneumonia in China, 2019. N Engl J Med, 2020. 382(8): p. 727-733.

2. Wang, , J. Tang, and F. Wei, Updated understanding of the outbreak of 2019 novel coronavirus (2019nCoV) in Wuhan, China. J Med Virol, 2020. 92(4): p. 441-447.

3. Rello, J., et al., Coronavirus Disease 2019 (COVID-19): A critical care perspective beyond China. Anaesth Crit Care Pain Med,

4. Huang, C., et al., Clinical features of patients infected with 2019 novel coronavirus in Wuhan, Lancet, 2020. 395(10223): p. 497-506.

5. Song, , et al., SARS-CoV-2 induced diarrhoea as onset symptom in patient with COVID-19. Gut, 2020.

6. Ng, T., et al., SARS-CoV-2 Infection among Travelers Returning from Wuhan, China. N Engl J Med, 2020.

7. Xu, Z., et al., Pathological findings of COVID-19 associated with acute respiratory distress syndrome. Lancet Respir Med, 
8. Lu, W., X.F. Liu, and Z.F. Jia, 2019-nCoV transmission through the ocular surface must not be ignored. Lancet, 2020. 395(10224):e39.

9. Sun, , et al., Understanding of COVID-19 based on current evidence. J Med Virol, 2020.

10. Ramsay, S. and M.A. Lerman, How to use the erythrocyte sedimentation rate in paediatrics. Arch Dis Child Educ Pract Ed, 2015. 100(1): p. 30-6.

11. Hanada, M., et al., Elevated erythrocyte sedimentation rate and high-sensitivity C-reactive protein in osteoarthritis of the knee: relationship with clinical findings and radiographic severity. Ann Clin Biochem, 2016. 53(Pt 5): p. 548-53.

12. Atzeni, , et al., Biomarkers in Rheumatoid Arthritis. Isr Med Assoc J, 2017. 19(8): p. 512-516.

13. Brouillard, M., et al., Erythrocyte sedimentation rate, an underestimated tool in chronic rena/ Nephrol Dial Transplant, 1996. 11(11): p. 2244-7.

14. Hess, T., Monitoring laboratory values: transferrin, C-reactive protein, erythrocyte sedimentation rate, and liver function. Adv Skin Wound Care, 2009. 22(2): p. 96.

15. Stojan, G., et al., Erythrocyte sedimentation rate is a predictor of renal and overall SLE disease Lupus, 2013. 22(8): p. 827-34.

\section{Tables}

Due to technical limitations, the tables are only available as a download in the supplemental files section.

\section{Figures}
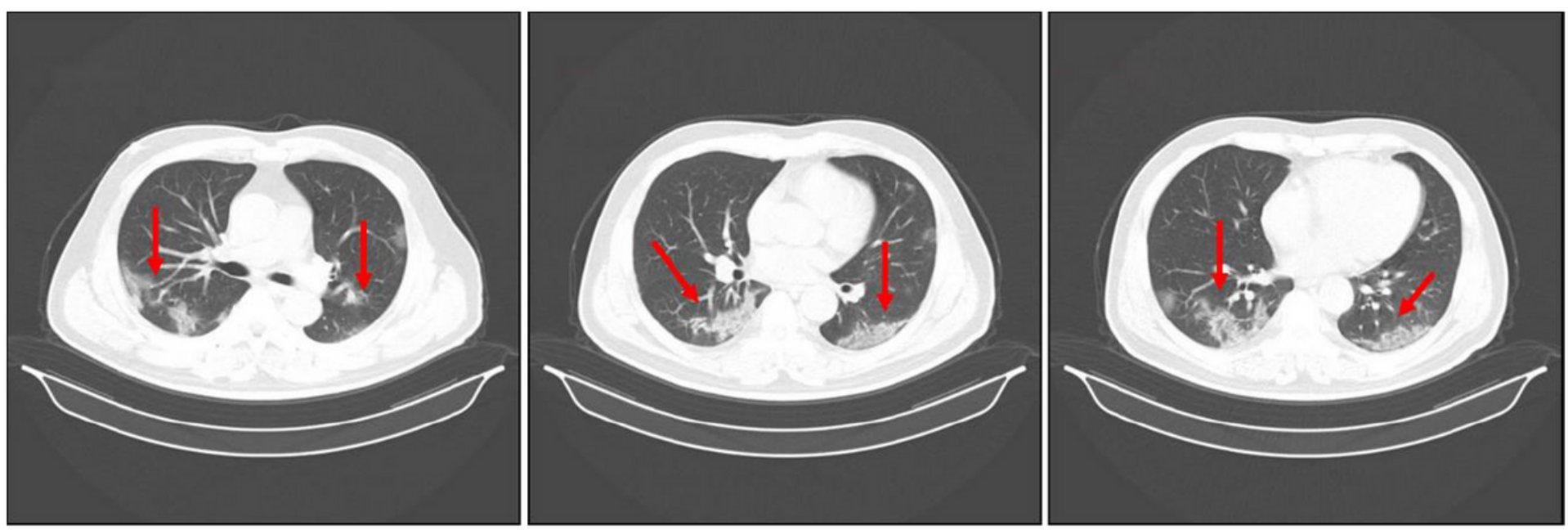

Figure 1

The images of chest CT scanned on January 31, 2020 

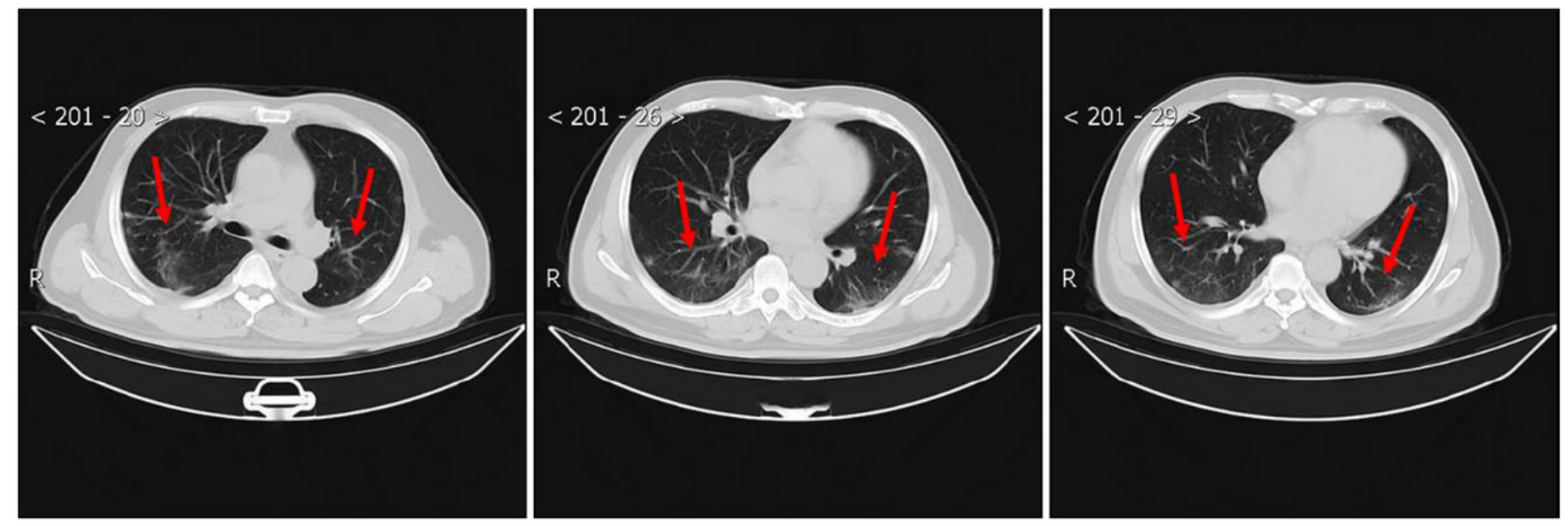

Figure 2

The images of chest CT scanned on February 15, 2020.
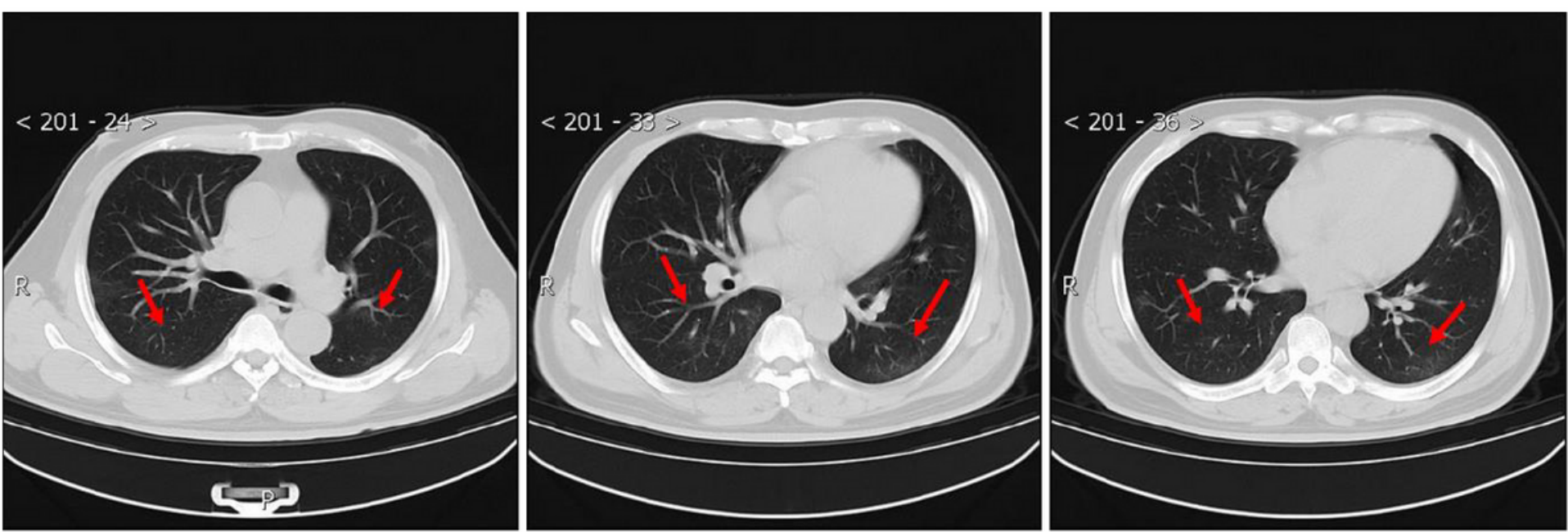

Figure 3

The images of chest CT scanned on February 25, 2020. 


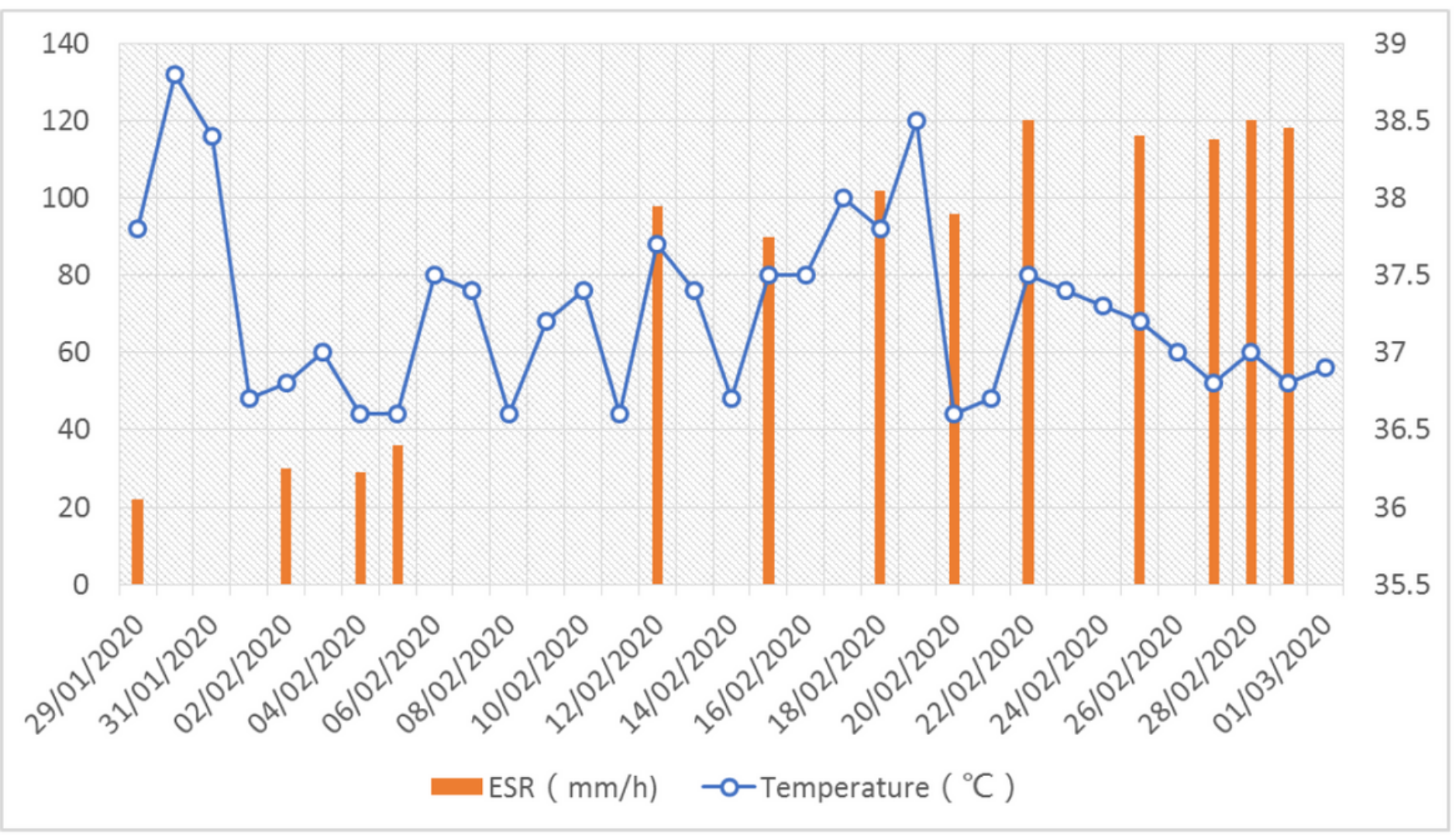

\section{Figure 4}

The dynamic curves of patient's body temperature and ESR during hospitalization.

\section{Supplementary Files}

This is a list of supplementary files associated with this preprint. Click to download.

- Table14SHB1.pdf 